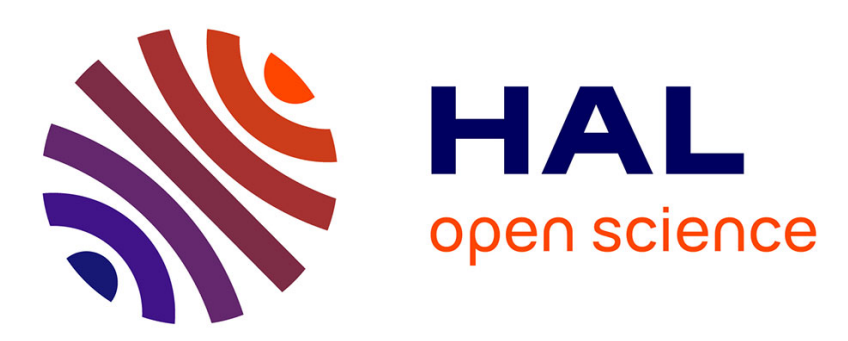

\title{
DVC-based image subtraction to detect microcracking in lightweight concrete
}

Camille Chateau, Thanh Tung T.T. Nguyen, Michel Bornert, Julien Yvonnet

\section{To cite this version:}

Camille Chateau, Thanh Tung T.T. Nguyen, Michel Bornert, Julien Yvonnet. DVC-based image subtraction to detect microcracking in lightweight concrete. Strain, 2018, 54 (5), pp.e12276. 10.1111/str.12276 . hal-01780698

\section{HAL Id: hal-01780698 \\ https://hal-enpc.archives-ouvertes.fr/hal-01780698}

Submitted on 20 Sep 2018

HAL is a multi-disciplinary open access archive for the deposit and dissemination of scientific research documents, whether they are published or not. The documents may come from teaching and research institutions in France or abroad, or from public or private research centers.
L'archive ouverte pluridisciplinaire HAL, est destinée au dépôt et à la diffusion de documents scientifiques de niveau recherche, publiés ou non, émanant des établissements d'enseignement et de recherche français ou étrangers, des laboratoires publics ou privés. 


\title{
DVC-based image subtraction to detect microcracking in lightweight concrete
}

\author{
C. Chateau ${ }^{a^{a}}$, T.T. Nguyen ${ }^{\mathrm{a}, \mathrm{b}}$, M. Bornert ${ }^{\mathrm{a}}$, J. Yvonnet $^{\mathrm{b}}$
}

\author{
a Laboratoire Navier, UMR 8205, CNRS, ENPC, IFSTTAR, Université Paris-Est, F-77455 Marne-la- \\ Vallée, France \\ ${ }^{b}$ Université Paris-Est, Laboratoire Modélisation et Simulation Multi-Echelle MSME UMR 8208 CNRS, 5 \\ bd Descartes, F-77454 Marne-la-Vallée, France
}

* Corresponding author: camille.chateau@enpc.fr - tel: +33164153662 - fax: +33164153741

\begin{abstract}
This paper presents an image subtraction technique based on Digital Volume Correlation (DVC) to detect and extract the complex network of microcracks that progressively developed in a lightweight concrete sample submitted in situ to uniaxial compression and imaged by X-ray computed tomography (XRCT). From local DVC measurements, performed only on positions with sufficient image contrast, the mechanical transformation is estimated at all voxels within the whole sample using an adjusted interpolation procedure that computes an affine approximation of the local transformation. The deformed image (containing cracks) is thus transformed back to the same frame as the reference image (without cracks) to compute the difference between both images, taking into account possible brightness and contrast adjustments. The resulting subtracted image reveals the path of cracks which is clearly visible without the underlying heterogeneous microstructure of the concrete. The detection accuracy is here estimated to one-tenth of a voxel, allowing early age cracks to be detected while they would barely have been noticed on the XRCT images. Segmentation of the crack network is also made much easier. To overcome a low signal-to-noise ratio for the tiniest cracks, a Hessian based filter is used to extract the complex crack network. The cracks can be directly located in the microstructure segmented in the reference image and compared for all loading steps to characterise their initiation and propagation.
\end{abstract}

Keywords: damage, digital volume correlation, in situ compression, lightweight concrete, x-ray tomography

NOTICE : This is the peer reviewed version of the following article: Chateau C, Nguyen TT, Bornert M, Yvonnet J. DVC-based image subtraction to detect microcracking in lightweight concrete. Strain. 2018;54:e12276, which has been published in final form at https://doi.org/10.1111/str.12276. This article may be used for non-commercial purposes in accordance with Wiley Terms and Conditions for Use of Self-Archived Versions. 


\section{Introduction}

The study of cracking occurring in many civil engineering materials, such as concrete or rocks, is of great concern for a better understanding of their mechanical properties and is essential in determining their reliability and durability. Detailed experimental characterisation is still needed for a better understanding of fracture mechanisms, in terms of initiation, propagation or localisation leading to macroscopic failure. Such data are also necessary to develop and to validate predictive models of crack nucleation and propagation at microscale ${ }^{[1]}$. The increasing use of X-Ray Computed Tomography (XRCT) in material science ${ }^{[2], ~[3]}$ makes it possible to characterise both microstructure and damage mechanisms in 3D within the bulk of materials. This non-destructive technique allows in situ experiments to be performed ${ }^{[4]}$ and then to characterise cracking evolution under load. In particular, cracks in cement-based materials could be observed e.g. under uniaxial ${ }^{[5-7]}$ and triaxial ${ }^{[8]}$ compression loading. Such materials may exhibit a complex cracking which can be difficult to fully characterise, especially because of their brittle nature involving low strains and narrow cracks that can propagate suddenly. In addition, these cracks may be difficult or even impossible to extract from the surrounding heterogeneous microstructure, especially at early age, which is required to perform a local and quantitative characterisation of fracture mechanisms. In particular, basic image analysis techniques, such as grey level thresholding, do not allow cracks to be

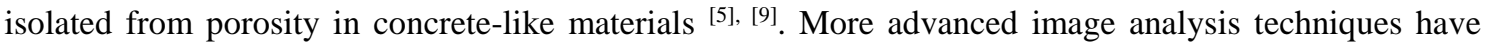
been considered to perform crack segmentation, such as ${ }^{[10-12]}$. However, even assuming the possibility of isolating cracks in a single XRCT image, the specimen deformation prevents direct comparison of successive scans, slice by slice, obtained from an in situ test. Damage characterisation regarding crack appearance and propagation is thus limited.

Combining XRCT in situ experiment to Digital Volume Correlation (DVC), which is a direct 3D extension of digital image correlation, is a way to overcome this restriction, by analysing in particular the correlation residuals. The latter correspond to remaining discrepancies between the mapped images essentially due to unavoidable image noise and to mismatch between the assumed kinematics optimized by the DVC routines and the real transformation which might be more complex ${ }^{[13], ~[14]}$. They essentially reveal discontinuities that are not described by the assumed mechanical transformation, corresponding to cracks for a brittle material. Thanks to the subvoxel accuracy of DVC measurement, a better detection sensitivity is expected compared to classical image processing ${ }^{[14]}$. The analysis of correlation residuals has been carried out from so-called global DVC measurements, in which the mechanical transformation is defined at all voxels of the region of interest, to study cracking mainly in metals ${ }^{[15-17]}$, but also in plaster ${ }^{[14]}$ and concrete-like material ${ }^{[18]}$.

This paper focuses on a similar approach where DVC is performed using local routines [19], [20]. It consists in computing the difference between the reference image and a deformed image exhibiting cracks, corrected by the transformation map estimated from local DVC measurements and an adjusted interpolation procedure to continuously extend the latter throughout the whole sample, even in areas where DVC would not work because of insufficient image contrast. This method is therefore adapted to strongly heterogeneous materials. It has been used to detect and extract cracks in a lightweight concrete sample under in situ compressive load in a XRCT laboratory scanner. Consisting of a cementitious matrix and large air voids, generated here by expanded polystyrene (EPS) beads, this kind of concrete exhibits in particular a low density and good thermal insulation and energy-absorbing properties ${ }^{[21-23]}$. Regarding in situ experimentation, this material has the advantage of showing crack initiation at relatively low compressive loads and stable crack propagation due to its high porosity. The specific experimental procedure for the in situ test is presented in section 2. Then, the DVC-based image subtraction is fully detailed in section 3 and the crack detection accuracy is discussed. Its use for the lightweight concrete images is presented in section 4. It is complemented by a processing of the obtained subtracted images to segment the cracks and to get a qualitative characterisation of the crack network regarding particularly initiation, morphology and propagation. 


\section{Experimental procedure}

\subsection{Material}

The material under study is an expanded polystyrene (EPS) lightweight concrete. It is made from quartz sand and EPS beads, whose typical sizes are $1 \mathrm{~mm}$ and $2 \mathrm{~mm}$ respectively, embedded in a cement matrix. A cylindrical specimen (11.7 $\mathrm{mm}$ in diameter and $19.9 \mathrm{~mm}$ long) was machined from a cubic sample using diamond core drilling and cutting. In order to avoid the initiation of damage from the ends of the sample due to parallelism imperfection and friction, both sides were polished with fine SiC paper. A maximum parallelism error of $30 \mu \mathrm{m}$ (difference in length at several positions) was obtained using the sample holder of an automatic polishing machine.

As shown by the XRCT slice in Figure 1, the microstructure is composed of three main phases: cement paste, sand grains and porosity. The latter is made of EPS beads and residual air voids of various sizes. Volume fractions have been estimated to $22 \%$ for porosity and $44 \%$ for quartz sand, after segmenting a XRCT image of the whole undamaged specimen.
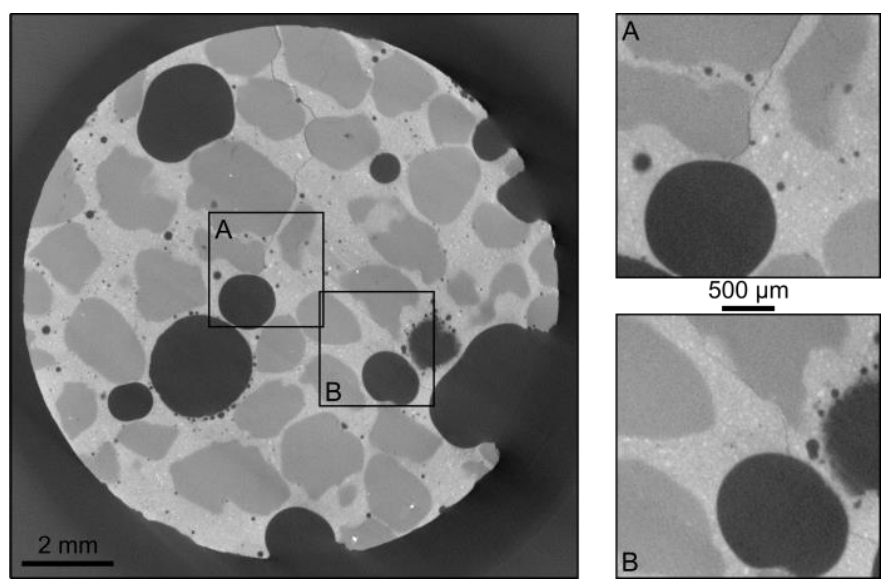

Figure 1: XRCT slice of the lightweight concrete sample under compressive load (18 MPa) and enlargements around cracks.

\subsection{In situ device}

In order to capture the evolution of the crack network under compressive loading, XRCT images were acquired at several damage levels. In order to avoid crack closure after releasing the load for scanning the specimen after each loading step (as would be done in an ex situ experiment), a specific in situ compression device has been used to hold the loading state during each scan (Figure 2a). The test was performed on the XRCT laboratory scanner available at Laboratoire Navier (Ultratom from RXSolutions), specifically designed to receive in situ experiments, thanks in particular to a hollow rotation stage able to support heavy samples (up to $100 \mathrm{~kg}$ ). The compression machine was designed and manufactured by LMS (Ecole polytechnique, France) and it is mainly made of steel to ensure the mechanical strength, except for a polycarbonate tube around the specimen where X-rays transparency is required. The load is prescribed to the specimen through aluminium rods with low X-ray attenuation to limit imaging artefacts at the boundaries. As shown in Figure $2 b$, two aluminium plates with same diameter as the sample are added at both ends of the specimen. In addition to their geometry designed to avoid the punching effect, these tabs have been carefully polished and silicon grease was added at their interface with the sample to reduce friction effect. Indeed, as described in more details in ${ }^{[24]}$, the transverse strain prescribed by the plates to the sample plays a central role on the initiation of damage near the ends of the sample. It depends on the discrepancy of the Poisson's ratio/Young's modulus ratios of the materials in contact and on the friction coefficient. In addition to the careful preparation of the sample, these precautions are essential to avoid early initiation of cracks due to imperfections of the boundary conditions. 

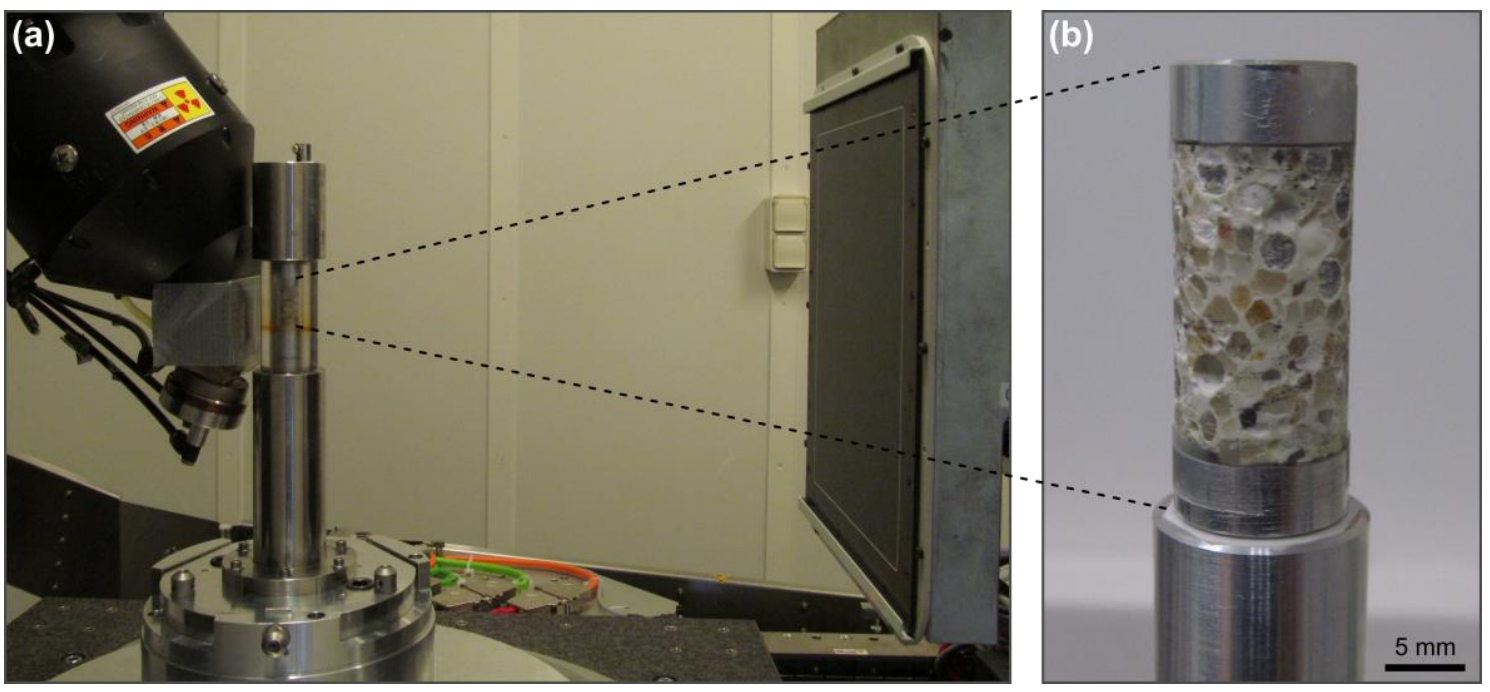

Figure 2: (a) Experimental device for compression test with XRCT imaging and (b) lightweight concrete sample with aluminium plates.

Note that an attempt to detect cracking during loading from acoustic emission was made using an USB AE Node system (Mistras) and a miniature sensor stuck at the upper end of the machine (see Figure 2a). Although a gradual evolution of $\mathrm{AE}$ has been noticed, in particular at the beginning of the test, one sensor is insufficient to ensure that hits were emitted from the sample. This will therefore not be discussed further here.

\subsection{XRCT imaging}

The XRCT images were captured using a micro-focus source Hamamatsu L10801 (max. tension $230 \mathrm{kV}$, max. power 200W, minimal spot size $5 \mu \mathrm{m})$ and a flat-panel imager Paxscan Varian 2520 V (1920x1536 pixels, pixel size $127 \mu \mathrm{m}$ ) depicted in Figure $2 \mathrm{a}$. The tube voltage and the tube current of the source have been set to $130 \mathrm{kV}$ and $78 \mu \mathrm{A}$ respectively. Two $360^{\circ}$ scans, each containing 1120 projections, were necessary to cover the whole sample height using a helical image acquisition mode, in which source and detector are moved vertically synchronously with the angular rotation of the sample. In order to improve signal to noise ratios of the projections, twelve radiographs recorded at the same angular position were averaged. The frame rate of the image sensor was set to 3 projections/sec. Altogether, these imaging conditions resulted is scan times of about 3 hours for a single 3D image with a voxel size of $10 \mu \mathrm{m}$. The reconstructed volumes contain about 1200x1200x2000 voxels and cover the whole sample. 3D images have been reconstructed by means of the X-Act software provided by the manufacturer of the XRCT device, which is based on the filtered back projections and the Feldkamp et al. algorithm for conical geometry ${ }^{[25]}$.

\subsection{Description of the compression test}

At the beginning of the test, a so-called reference image was acquired under a preload of a few Newtons. A second image, called zoomed image, was acquired after a slight magnification change $(\sim 0.5 \%)$ obtained with a little move of the detector. This image will serve to quantify image correlation performances and in particular define the set of correlation points with a suitable local image contrast, as shown in section 4.1.1. Then, twelve successive loading steps were applied to the specimen up to $1.94 \mathrm{kN}$ (18 MPa), with very slow displacement rates $\left(2.10^{-4} \mathrm{~mm} / \mathrm{s}\right.$ for the first step and $10^{-4} \mathrm{~mm} / \mathrm{s}$ for the others) to ensure a slow and stable propagation of cracks. After each loading step, the displacement was kept constant until the next loading and a XRCT image was acquired after a relaxation time lasting a few minutes. Note that the whole test lasted several days and was carried out in only one loading phase, without intermediate 
unloading.

First cracks were observed inside the sample from the third step ( 9 MPa). Then the crack network progressively developed and propagated to most of the sample. As depicted in Figure 1, several cracks can be directly observed in the XRCT images. However, because their grey level is very similar to that of the porosity and close to that of other constituents, their extraction from XRCT images with conventional segmentation tools is not straightforward. Moreover, most of them are only slightly open, with respect to the voxel size, making them hard to detect, especially in their early stage.

\section{DVC-based image subtraction}

\subsection{Digital Volume Correlation: background}

Local DVC analyses ${ }^{[26]}$ were performed on the images, using the in-house software CMV_3D developed at Laboratoire Navier [19], [20]. The procedure is a straightforward extension to 3D images of local digital image correlation. It consists in finding homologous positions of a set of material points in two images, considering the grey level distribution in a small subvolume around each point ${ }^{[13]}$. Main features and notations are reminded below.

Let $f$ and $g$ be the three-dimensional grey levels in reference and deformed digital images respectively. Let $\phi$ be the mechanical transformation that links the position $\boldsymbol{X}$ in reference image to the position $\boldsymbol{x}$ in the deformed image such as $\boldsymbol{x}=\boldsymbol{\phi}(\boldsymbol{X})$. The evaluation of the exact voxel wise transformation $\phi$ from the knowledge of $f$ and $g$ is an ill-posed problem, because grey levels of voxels do not provide a sufficient information. Therefore, it is approximated by much simpler transformation defined by a very limited number of parameters. In the simplest case, here adopted, it is defined as a pure uniform translation in the subvolume $D$, also called correlation domain, centred on the position $\boldsymbol{X}_{\mathbf{0}}$, also called correlation point:

$$
\phi_{0}(\boldsymbol{X})=\boldsymbol{X}+\boldsymbol{u}\left(\boldsymbol{X}_{\mathbf{0}}\right), \text { for all } X \text { in } D
$$

where $\boldsymbol{u}$ is the displacement field. This selection of local transformation is satisfactory when the actual deformation gradient is small and one uses small correlation domains. The associated shape function mismatch errors ${ }^{[27]}$ might then be of the order of a few hundredths of a voxel, and noticeably lower than errors due to image noise. The three components of the translation for each correlation domain are identified as those which minimize the discrepancy between reference and deformed configurations, measured by the so-called correlation coefficient. The latter measures the similarity, in terms of grey level distribution, between the domain $D$ and the corresponding domain $\phi_{0}(D)$ by computing the so-called zero centred normalized cross-correlation coefficient:

$$
C\left(\phi_{0}\right)=1-\frac{\sum_{\boldsymbol{X} \in D}\left[f(\boldsymbol{X})-\bar{f}_{D}\right] \cdot\left[g\left(\phi_{0}(\boldsymbol{X})\right)-\bar{g}_{D}\right]}{\sqrt{\sum_{\boldsymbol{X} \in D}\left[f(\boldsymbol{X})-\bar{f}_{D}\right]^{2} \cdot \sum_{\boldsymbol{X} \in D}\left[g\left(\phi_{0}(\boldsymbol{X})\right)-\bar{g}_{D}\right]^{2}}}
$$

where $\bar{f}_{D}$ and $\bar{g}_{D}$ are the averages of grey levels $f$ and $g$ in $D$ and $\phi_{0}(D)$ respectively. This formulation is insensitive to global contrast or brightness variations on the domains and it leads to values of $C\left(\phi_{0}\right)$ from 0 (perfect match) to 2 (anti-correlation). A continuous trilinear interpolation of grey levels is used to compute $g\left(\phi_{0}(\boldsymbol{X})\right)$ and its gradients since the components of $\boldsymbol{u}\left(\boldsymbol{X}_{\mathbf{0}}\right)$ do not necessarily take integer values (in voxel units).

In practice, in the used CMV_3D software, the optimization of the local translation components is split into two steps in order to increase the computational efficiency of the DVC routines. First, sets of discrete translations (i.e. with components equal to integer numbers of voxels) are systematically explored for the best correlation coefficient. This exploration can easily be parallelized on a multi-core computer. Then, 
subvoxel accuracy is searched for using an appropriate iterative optimization algorithm. While this optimization is harder to parallelise efficiently, it is easy to distribute the independent optimization tasks for each correlation point over several CPU-cores. This optimization is only performed for points at which the correlation coefficient obtained after the first step is below a user-selected threshold.

\subsection{Image subtraction}

\subsubsection{General principle}

The sparse evaluation of the transformation map $\phi$, obtained by DVC, can be continuously extended throughout the whole sample by a dense approximation $\phi_{1}(\boldsymbol{X})$, as described in next section. Because the position $\phi_{1}(\boldsymbol{X})$ is not always an integer, the grey level $g\left(\phi_{1}(\boldsymbol{X})\right)$ in the deformed image, corresponding to any voxel $\boldsymbol{X}$ with grey level $f(\boldsymbol{X})$ in the reference image, can be obtained using either trilinear or tricubic interpolation. Thus, the deformed image is transformed back to the same frame as the reference image. Finally, the difference between reference and deformed images defines the subtracted image as

$$
r(\boldsymbol{X})=g\left(\phi_{1}(\boldsymbol{X})\right)-f(\boldsymbol{X})+k
$$

where the offset $k$ ensures a positive grey level of the subtracted image and is taken as $k=128$ for 8 -bit greyscale images.

The subtracted image reflects the local evolutions within the material, not described by the fit of the coarse evaluation of the transformation provided by DVC measurements and their interpolation. For a brittle material, it essentially gives access to the cracks: the subtracted images mainly consist in an uniformly grey background, with an average grey level $k$, in which the crack network appears in darker grey. Note that within areas with sufficient local contrast where DVC routines are run successfully, the subtracted image essentially coincides with standard so-called correlation residuals ${ }^{[13]}$, even if is computed at a later stage of the processing. The presented procedure extends the latter approach to the situation of a non-uniform distribution of contrast.

This definition of the image subtraction only applies in case of perfect grey level conservation. This was achieved here by using rigorously identical imaging procedures and settings for all loading steps, as well as the same grey level range when converting the format of the original XRCT images (32-bit floats representing attenuation coefficients) to the used one (8-bit). Otherwise, Eq.(3) could easily be extended to allow for a possible variation in image contrast and brightness, respectively corrected by coefficients $a$ and $b$ :

$$
r(\boldsymbol{X})=\left[a \cdot g\left(\phi_{1}(\boldsymbol{X})\right)+b\right]-f(\boldsymbol{X})+k,
$$

where

$$
a=\frac{\sum_{\boldsymbol{X} \in \Omega}\left[f(\boldsymbol{X})-\bar{f}_{\Omega}\right] \cdot\left[g\left(\phi_{1}(\boldsymbol{X})\right)-\bar{g}_{\Omega}\right]}{\sum_{\boldsymbol{X} \in \Omega}\left[g\left(\phi_{1}(\boldsymbol{X})\right)-\bar{g}_{\Omega}\right]^{2}}
$$

and

$$
b=\bar{f}_{\Omega}-a \bar{g}_{\Omega} .
$$

Both coefficients could be computed at each voxel in the calculation domain $\Omega$ centered on $\boldsymbol{X}$, the size of which could be equivalent to that of the correlation domain $D$ or of the vicinity used to define the interpolated local transformation (defined in section 3.2.2). To avoid additional computation cost, they can also be supposed to be constant and computed only once using a larger domain $\Omega$. Several pairs of 
coefficients might also be used for contrasted areas of the images (see ${ }^{[24]}$ for details).

\subsubsection{Local transformation approximation}

As discussed in the previous section, the full determination of the local transformation $\phi$ at each voxel is an ill-posed problem. This work aims at constructing the approximation $\phi_{1}$ of the local transformation by using a dense continuation of the transformation of nearby correlation points. After the DVC procedure, only the positions of well-correlated correlation points are retained, in both reference image and deformed images. Well-correlated points are correlation points whose correlation coefficient is below a userselected value, which will be discussed for the lightweight concrete sample in section 4.1.1.

Let $\boldsymbol{X}$ be the position of the voxel under consideration in the reference image ( $\boldsymbol{X}$ is supposed to be the centre of the voxel) and $\boldsymbol{X}_{\mathbf{0}}$ the position of its nearest neighbour correlation point with measured displacement $\boldsymbol{u}\left(\boldsymbol{X}_{\mathbf{0}}\right)$. Using a first-order expansion of the displacement field near point $\boldsymbol{X}_{\mathbf{0}}$, we set:

$$
\phi_{1}(\boldsymbol{X})=\boldsymbol{X}+\boldsymbol{u}\left(\boldsymbol{X}_{\mathbf{0}}\right)+\frac{\partial \boldsymbol{u}}{\partial \boldsymbol{X}}\left(\boldsymbol{X}_{\mathbf{0}}\right)\left(\boldsymbol{X}-\boldsymbol{X}_{\mathbf{0}}\right)
$$

In three dimensions, this global transformation is locally defined by 12 parameters, including 3 components of the displacement and 9 components of the displacement gradient at $\boldsymbol{X}_{\mathbf{0}}$. By definition of the deformation gradient $\boldsymbol{F}=\frac{\partial \phi}{\partial \boldsymbol{X}}$, we also have:

$$
\frac{\partial \boldsymbol{u}}{\partial \boldsymbol{X}}\left(\boldsymbol{X}_{0}\right)=\boldsymbol{F}\left(\boldsymbol{X}_{0}\right)-\boldsymbol{I}
$$

where $\boldsymbol{I}$ is the identity tensor. An estimate of $\boldsymbol{F}\left(\boldsymbol{X}_{\mathbf{0}}\right)$ is therefore needed to determine $\phi_{1}$.

To do so, it is proposed to consider the affine best fit of the DVC measured displacements of $N$ neighbour well-correlated correlation points of the considered point $\boldsymbol{X}$. Let $\phi_{N}$ be this transformation. It is given by:

$$
\phi_{N}(X)=T_{N}(X)+F_{N}(X) \cdot X
$$

where the second-order tensor $\boldsymbol{F}_{\boldsymbol{N}}$ and the translation vector $\boldsymbol{T}_{\boldsymbol{N}}$ are obtained from a classical least square fitting procedure defined by:

$$
\boldsymbol{T}_{N}, \boldsymbol{F}_{\boldsymbol{N}}=\operatorname{Argmin} \sum_{i=1}^{N}\left\|\boldsymbol{x}_{\boldsymbol{i}}-\left(\boldsymbol{T}_{\boldsymbol{N}}+\boldsymbol{F}_{\boldsymbol{N}} \cdot \boldsymbol{X}_{\boldsymbol{i}}\right)\right\|^{2}
$$

where $\boldsymbol{x}_{\boldsymbol{i}}=\phi\left(\boldsymbol{X}_{\boldsymbol{i}}\right)$ is the DVC-estimated position in deformed configuration of the neighbouring correlation point $\boldsymbol{X}_{\boldsymbol{i}}$ in reference configuration. These optimal translation and deformation gradient are thus given by:

$$
\boldsymbol{T}_{\boldsymbol{N}}=\langle\phi(\boldsymbol{X})\rangle_{N}-\boldsymbol{F}_{\boldsymbol{N}} \cdot\langle\boldsymbol{X}\rangle_{N}
$$

and

$$
\boldsymbol{F}_{\boldsymbol{N}}=\left[\sum_{i=1}^{N}\left(\boldsymbol{x}_{\boldsymbol{i}}-\langle\boldsymbol{x}\rangle_{N}\right) \otimes\left(\boldsymbol{X}_{\boldsymbol{i}}-\langle\boldsymbol{X}\rangle_{N}\right)\right]\left[\sum_{i=1}^{N}\left(\boldsymbol{X}_{\boldsymbol{i}}-\langle\boldsymbol{X}\rangle_{N}\right) \otimes\left(\boldsymbol{X}_{\boldsymbol{i}}-\langle\boldsymbol{X}\rangle_{N}\right)\right]^{-1}
$$

where $\langle a\rangle_{N}$ is the average of the $N$ values of the quantity a computed for the $N$ well correlated neighbour points $\boldsymbol{X}_{\boldsymbol{i}}, i \in[1, N]$. 
Using this affine local best fit, the dense continuation $\phi_{1}$ of the mechanical transformation can then be defined at any point $\boldsymbol{X}$ in the sample by:

$$
\phi_{1}(X)=X+u\left(X_{0}\right)+\left(F_{N}(X)-I\right) \cdot\left(X-X_{0}\right)
$$

An alternative could be to define it as the affine best fit itself, i.e. $\phi_{1}(\boldsymbol{X})=\phi_{N}(\boldsymbol{X})$ as defined in Eq.(9). The results presented in the following section have been obtained with this second option.

The first option provides a more local dense estimation of the displacement field, which strictly coincides with the DVC-estimated transformation at well-correlated point of the DVC grid, but is for this reason much more sensitive to the DVC errors on the displacement of the sole nearest neighbour correlation point. The second option is less sensitive to correlation errors, because they are averaged over $N$ positions and leads to a smoother fitted displacement field which might not coincide strictly with DVC measurements. Note that both options generate transformations which are piecewise affine since $\boldsymbol{T}_{\boldsymbol{N}}(\boldsymbol{X})$ and $\boldsymbol{F}_{\boldsymbol{N}}(\boldsymbol{X})$ are piecewise constant over sets of points $\boldsymbol{X}$ which share the same well-correlated neighbour points. There might be some kinematic discontinuities at the borders of these sets, which are however of limited amplitude, especially when the second option is used, since only a limited part of the DVC measurements used to perform the fit is modified at such interfaces.

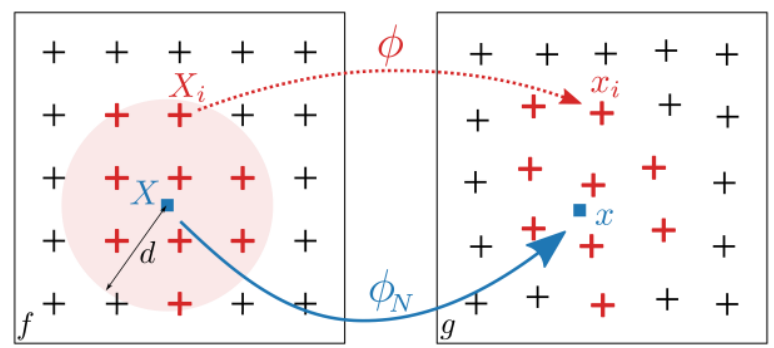

Figure 3: Schematic representation of the continuous approximation of the local transformation from neighbour correlation points.

As illustrated in Figure 3, the neighbour correlation points are selected according to a criterion of maximum distance $d$ from the considered voxel $\boldsymbol{X}:\left\|\boldsymbol{X}-\boldsymbol{X}_{\boldsymbol{i}}\right\| \leq d$, where $\|$. $\|$ is the Euclidean norm. The neighbour transformation depends on this criterion. A small distance gives a more "local" transformation which may reflect more accurately local strain heterogeneities or discontinuities in the material, but the obtained result is more sensitive to DVC errors. These errors are smoothed out when a larger distance is used, which however provides a less local observation, and requires more computation time. This effect is also related to the distance between correlation points. A large distance, associated with a large number of neighbours, will also generate an almost continuous dense transformation $\phi_{1}$. A strictly continuous transformation could be generated if the affine fit would be performed with weights attributed to the neighbour correlation points $\boldsymbol{X}_{\boldsymbol{i}}$ continuously decreasing to zero when the distance $\left\|\boldsymbol{X}-\boldsymbol{X}_{\boldsymbol{i}}\right\|$ gets close to $d$. Such a mathematical sophistication is in practice however of limited interest and would generate higher computational cost.

In practice, the selection of the set of neighbour points of each voxel in the reference image is a computationally very expensive task, if a basic algorithm which would test all correlation points in the DVC mesh is used. To reduce the computation time, the distance test has been restricted to points which are known to be close to the considered voxel from an initial classification of all correlation points. Moreover, the computation of $\boldsymbol{F}_{\boldsymbol{N}}$ requires the inversion of the matrix $\boldsymbol{M}$ defined by (see Eq. (12)):

$$
\boldsymbol{M}=\sum_{i=1}^{N}\left(\boldsymbol{X}_{\boldsymbol{i}}-\langle\boldsymbol{X}\rangle_{N}\right) \otimes\left(\boldsymbol{X}_{\boldsymbol{i}}-\langle\boldsymbol{X}\rangle_{N}\right)
$$


$\boldsymbol{M}$ is symmetric and positive because it is the sum of rank-one tensors of the form $\boldsymbol{A}_{\boldsymbol{i}} \otimes \boldsymbol{A}_{\boldsymbol{i}}$. However, it might not be definite if the span of the vectors $\boldsymbol{A}_{\boldsymbol{i}}$ does not cover the whole 3D space. This is in particular the case when all neighbour correlation points are distributed in a same plane. The out-of-plane components of the transformation gradients can then not be defined. More generally, when points are close to be co-planar, these out of plane components will not be accurately determined. In the used implementation of the procedure, a criterion is thus adopted to check the singularity of $\boldsymbol{M}$ based on the analysis of its eigenvalues. It requires that the ratio of the smallest to the largest eigenvalue is not too small (eigenvalues are computed with the QL algorithm proposed by ${ }^{[28]}$ ). As long as the number of neighbour correlation points is insufficient to meet the criterion, the maximal distance $d$ is automatically increased to select more correlation points, according to a distance step chosen by the user. Beyond a given maximal distance, the user can also choose to make use of the global transformation (computed as the fit of all correlation point in the reference image). Such cases mainly arise in regions with little or no contrast, e.g. in EPS beads or big sand grains for the present application. In such areas, an inaccurate estimation of the transformation has little impact on the subtracted image.

Let us finally emphasize on the fact that the here proposed methodology differs from the computation of the so-called correlation residuals in the context of global DVC algorithms, by the fact that the global transformation $\phi_{1}$ used to subtract the images differs from the local transformations $\phi_{0}$ used to run DVC. This might be seen as an inconsistency of the proposed methodology; it might also be seen as an additional advantage, through the opportunity it provides to freely define the scale of variations of the interpolated kinematic field, in particular through the choice of the distance $d$, which might be very different from the size of the correlation domains.

\subsubsection{Estimation of crack detection accuracy}

This section aims at roughly estimating the smallest opening of a crack, which allows one to distinguish it from the noise in the subtracted image. As illustrated in Figure 1, the presence of a crack in the deformed image results in a drop of grey levels in the crack, which may go down to the grey level in porosity (i.e. in air) for the largest ones (whose opening is larger than one voxel). We consider a grey level profile crossing a crack in the deformed image. Let $\Delta_{g}$ be the difference between the average grey level of the solid phase $\bar{g}_{s o l}$ and the lowest grey level reached in the crack $g\left(\boldsymbol{x}_{\boldsymbol{c}}\right)$. In perfect absorption contrast tomographic images, the grey level of a voxel is an affine function of the effective linear X-ray attenuation coefficient of the material contained in the physical volume associated with the voxel in the real sample. When this material is heterogeneous, the effective attenuation coefficient is an average of the attenuations of the constitutive pure phases, weighted by their volume fraction. This is the case of a crack passing through this physical volume, because it is then made of void (or air) and surrounding solid phase (which itself might be multiphasic). As a consequence, the grey level of a voxel crossed by cracks evolves from the grey level of pure solid phase to the grey level of pure air, as a linear function of the proportion of void in the physical volume associated with the voxel. In the case of a crack parallel to the axes of the $3 \mathrm{D}$ image, this proportion is equal to the opening of the crack when the latter is smaller than one voxel. Hence, we have:

$$
\left\{\begin{array}{l}
\Delta_{g}=\Delta_{\max }, \text { if } e>1 \\
\Delta_{g}=e \Delta_{\max }, \text { if } e \leq 1
\end{array}\right.
$$

where $\Delta_{\max }$ is the difference between $\bar{g}_{s o l}$ and the average grey level of the porous phase $\bar{g}_{\text {por }}$, and $e$ is the crack opening in voxel unit. When the crack is not parallel the image axes, this relation might be modified by some geometric parameters, which will however not modify the essence of this relation.

The crack leads to a similar variation of grey levels in the subtracted image, denoted $\delta$, but less sharp because of interpolation effects. In fact, we have 


$$
\delta=\bar{g}_{s o l}-g\left(\phi_{1}\left(\boldsymbol{X}_{c}\right)\right)
$$

with $\boldsymbol{X}_{\boldsymbol{c}}$ being in the position in reference configuration such as $\phi_{1}\left(\boldsymbol{X}_{\boldsymbol{c}}\right)$ is the position closest to the crack position $\boldsymbol{X}_{\boldsymbol{c}}$ in the deformed image. If $\phi_{1}\left(\boldsymbol{X}_{\boldsymbol{c}}\right)$ has integer components, no interpolation is required, leading to $\delta=\Delta_{g}$. On the contrary, a trilinear interpolation is estimated to lead to $g\left(\phi_{1}\left(\boldsymbol{X}_{c}\right)\right)=\bar{g}_{\text {sol }}-$

$\frac{\Delta_{g}}{2}$, for the most unfavorable case where the voxel in the reference configuration is associated with half integer components in the deformed one. Hence, we get

$$
\frac{\Delta_{g}}{2} \leq \delta \leq \Delta_{g}
$$

For tricubic interpolation, the lower bound in this relation might be slightly modified but without affecting its order of magnitude.

From Eq. (15), an observed grey level drop $\delta$ in the subtracted image can thus be associated to a crack with an opening bounded by:

$$
\frac{\delta}{\Delta_{\max }} \leq e \leq \frac{2 \delta}{\Delta_{\max }}
$$

Thus, considering that the crack cannot be distinguished in the subtracted image if the grey level drop is smaller than the standard deviation $\sigma_{s}$ of the noise in the undamaged phase in the subtracted image (i.e. $\delta \geq \sigma_{s}$ is necessary for the crack to be detectable), the range restricting the minimum crack opening that may be detected $e_{\min }$ is estimated as

$$
\frac{\sigma_{s}}{\Delta_{\max }} \leq e_{\min } \leq \frac{2 \sigma_{s}}{\Delta_{\max }}
$$

Note that, assuming similar and statistically independent noise in the reference and deformed images, with standard deviation $\sigma_{f}$, the standard deviation of the noise in the subtracted image is equal to $\sigma_{s}=$ $\sqrt{2} \sigma_{f}$.

\section{Qualitative characterisation of microcracking in lightweight concrete}

\subsection{Implementation settings}

\subsubsection{DVC}

A fine mesh of correlation points is required to track local evolutions of the material and an appropriate local contrast is also necessary to ensure the DVC routines can run. However, the grey levels in porosity and sand grains are rather uniform. Then, local DVC routines were carried out in cement matrix only, and in a close neighbourhood of the sand grain interfaces. To do so, a fine regular grid was defined over the whole sample with an inter-point distance equal to 18 voxels. The positions of its points were then compared with the segmented image of sand grains (binary image with voxels in sand set to 1) which has been slightly shrunk by a morphological erosion: points whose surrounding average grey level was equal to 1 were excluded. Conversely, a similar process was performed to exclude points in pores or too close to pores, using a morphological dilation of the segmented porous phase. Hence, points located at pore interfaces are excluded but those at sand interfaces are retained. The size of the structural spherical element of the morphological operations and that of the surrounding windows were set to 18 and 40 voxels respectively. A part of the obtained grid is shown in Figure 4. 


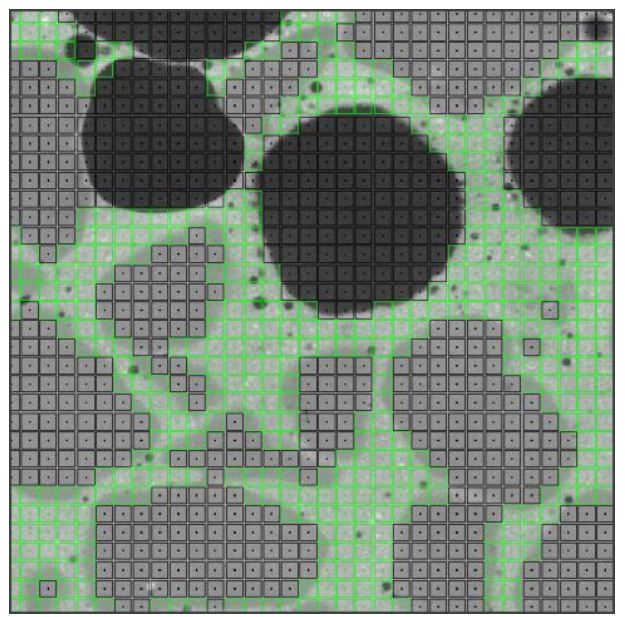

Figure 4: 2D view of the grid of correlation points in reference image. Retained points are coloured in green.

As explained in section 3.1, the correlation procedure was performed in two successive steps. The first correlation (to the nearest voxel) was run using a cubic correlation domain with 40 voxels on a side and a correlation coefficient criterion set to 0.3. For the following subvoxel correlation, using the same correlation domain size, a more restrictive correlation coefficient was used to select the more reliable points. The later has been defined from the analysis of the distribution of correlation coefficients obtained through the DVC analysis between the reference image and the zoomed image (restricted to selected points as defined above). The distribution, presented in Figure 5, shows two major peaks. The first narrow peak mostly corresponds to points located close to porosity with a high grey level contrast. The other one corresponds to points located in cement paste and at cement/sand interfaces where the image contrast is less pronounced. The points with a correlation coefficient in the distribution tail were excluded from the set of well correlated points, because their associated image contrast was considered to be insufficient, and potentially leading to larger DVC errors. In practice, they were selected by defining a correlation coefficient threshold $C_{0}=\langle C\rangle+\sigma(C), \sigma$ standing for the standard deviation, i.e. $C_{0}=0.07$.

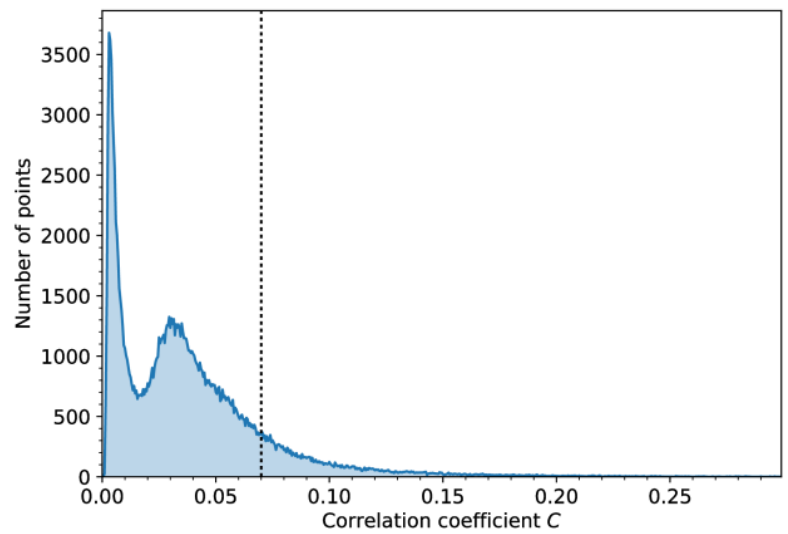

Figure 5: Distribution of correlation coefficients resulting from the DVC between the reference image and the zoomed image after subvoxel optimization.

DVC errors have been analysed from the measured displacements in the zoomed image satisfying the correlation threshold $C_{0}$. The imposed magnification variation leads to the theoretical displacement gradient: $F_{x x}-1=F_{y y}-1=F_{z z}-1=-4.816 \times 10^{-3}$. The best-fitting displacement gradient obtained by DVC on the whole sample was

$$
\boldsymbol{F}-\boldsymbol{I}=\left[\begin{array}{ccc}
-4.997 & 0.146 & 0.018 \\
-0.144 & -5.005 & 0.032 \\
-0.064 & 0.053 & -4.838
\end{array}\right] \times 10^{-3}
$$


which is in good agreement with the prescribed value, with a maximum discrepancy of $1.9 \times 10^{-4}$. The local displacement as measured by DVC can also be compared to the theoretical motion associated with the best-fitting affine deformation. This allows to evaluate the so-called random and systematic errors ${ }^{[29]}$ through the procedure presented in ${ }^{[30]}$, extended here to 3D. A maximal amplitude of systematic errors of 0.1 voxels and a random error in most cases of the order of 0.05 voxels, and reaching 0.15 voxels for displacement components close to integer values have been observed. These values will not be referred to later on in this paper, but may serve to quantify the typical DVC accuracy achieved for the here presented situation, for which the image subtraction procedure could be efficiently run.

\subsubsection{Image subtraction}

The local transformation was estimated from neighbour correlation points within the maximum distance $d=40$ voxels. In case of not enough points to compute the transformation (i.e. less than 4 ) or to satisfy the singularity criterion (matrix $\boldsymbol{M}$, see section 3.2.2), the distance was extended to $d=80$ voxels. This distance was still insufficient for about $16 \%$ of the volume of interest (including $1 \%$ of the volume failing the singularity criterion), mostly located in large pores or sand grains, for which image subtraction was performed using the global transformation. When the local transformation could be determined, 33 neighbour points were used in average (22 points when $d=40$ and 60 points when $d=80$ ) and the distance had to be increased for about $30 \%$ of voxels. The subtraction procedure, implemented in the CMV_3D software, was performed for each loading step in about 17 hours running on a workstation equipped with two hexa-core Intel Xeon X5680 CPU (3.33 GHz) and 64 Go RAM. This computational cost is mainly due to the search of neighbour correlation points when estimating the local transformation. It is highly dependent on the maximum neighbour distance and the number of correlation points. It might be reduced with additional efforts on the neighbour search algorithm.

Both tricubic and trilinear interpolation of grey levels have been tested. Results are compared in Figure 6 in a slice containing no crack in the deformed state. This example shows remaining interfaces in the subtracted image when using the trilinear interpolation (Figure 6c), especially pore/cement interfaces which are highly contrasted. The interpolation is here not sufficient to correct the possible shift, between reference and transformed image, of interfaces blurred due to the discretisation. Subtracted images have thus been computed using the tricubic interpolation, which seems to smooth out more efficiently these interfacial artefacts and mostly results in image noise only in undamaged regions (Figure 6b).

Note however, that cracks may also be smoothed out this way. A possible way to circumvent this and to enhance the crack detection accuracy would be possible to invert reference and deformed configurations to compute the subtracted image and thus to interpolate grey levels of the undamaged image. This solution has however not been adopted because the subtracted images would be built in the frame of the damaged configuration, which prevents the direct comparison, in particular in the exact same 2D observation planes, of the successive loading stages.
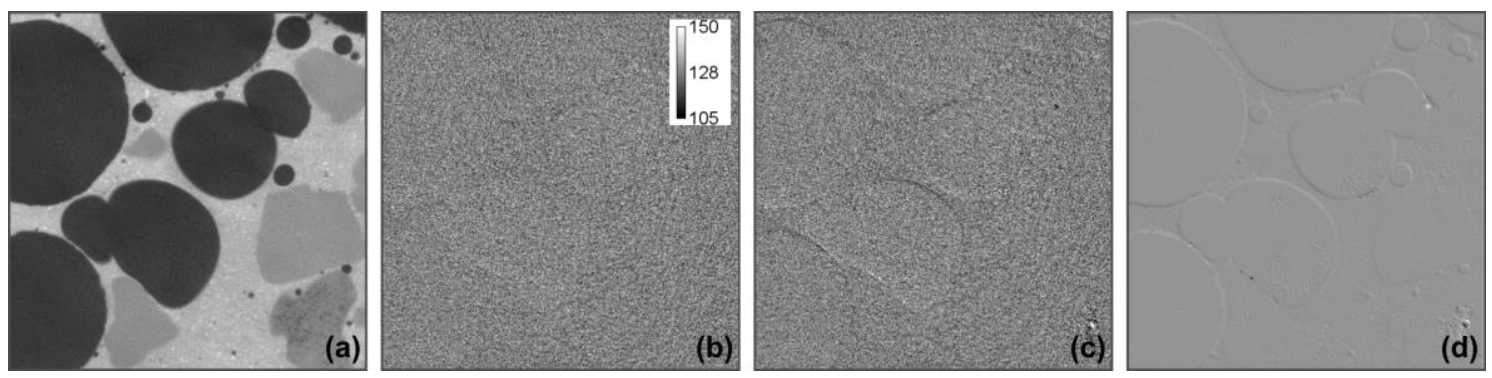

Figure 6: Effect of grey level interpolation at interfaces: (a) reference image, subtracted image using (b) tricubic or (c) trilinear interpolation. Contrast of (b) and (c) has been enhanced to highlight differences between both interpolations (according to the displayed greyscale bar). The latter are also illustrated in (d) which corresponds to the subtraction between (b) and (c) before contrast enhancement. 
Finally, this set of parameters leads to an image noise in the undamaged phase of subtracted images with a standard deviation of $\sigma_{s} \approx 5$. Note that the noise level is similar in areas corresponding to cement paste and sand grains. The maximum grey level gap $\Delta_{f}^{\max }$ in reference image has been estimated to 90 between sand grains and porosity. It rises to 120 in cement paste. Hence, following Eq. (19), the minimum crack opening that may be detected is estimated to $e_{\min }=0.11$ voxel (i.e. $1.1 \mu \mathrm{m}$ ) in sand grains in the worst case scenario. The accuracy is slightly better in cement paste with $e_{\min }=0.08$ voxel (i.e. $0.8 \mu \mathrm{m}$ ).

\subsection{Detection and segmentation}

An example of result, obtained with the method described above, is presented in Figure 7. As illustrated in Figure 7c, the subtracted image reveals cracks that are qualitatively observable in the deformed image, without the underlying microstructure. In addition, the subtraction method allows to select the newly created cracks or the evolving ones only. For example, cracks in pre-cracked sand grains are eliminated (if they do not evolve). Moreover, we can also observe tiny cracks that are not visible in the deformed image, such as those pointed by arrows. The path of the largest cracks is clearly visible and it could be segmented by a simple threshold of grey levels in the subtracted image. But for the smallest openings, the signal-to-noise ratio is too low to allow direct thresholding to extract cracks. This corresponds to the limit case addressed in section 3.2.3, where the grey level variation generated by the cracks is similar to the image noise. To unambiguously follow qualitatively the damage evolution during the compression test and compare it to the microstructure of the material, an alternative technique could be used as in ${ }^{[1]}$. It consists in only transforming the deformed images back to the reference frame, without performing the subtraction. The so-called transformed image would be defined as $t(\boldsymbol{X})=g\left(\phi_{1}(\boldsymbol{X})\right)$.
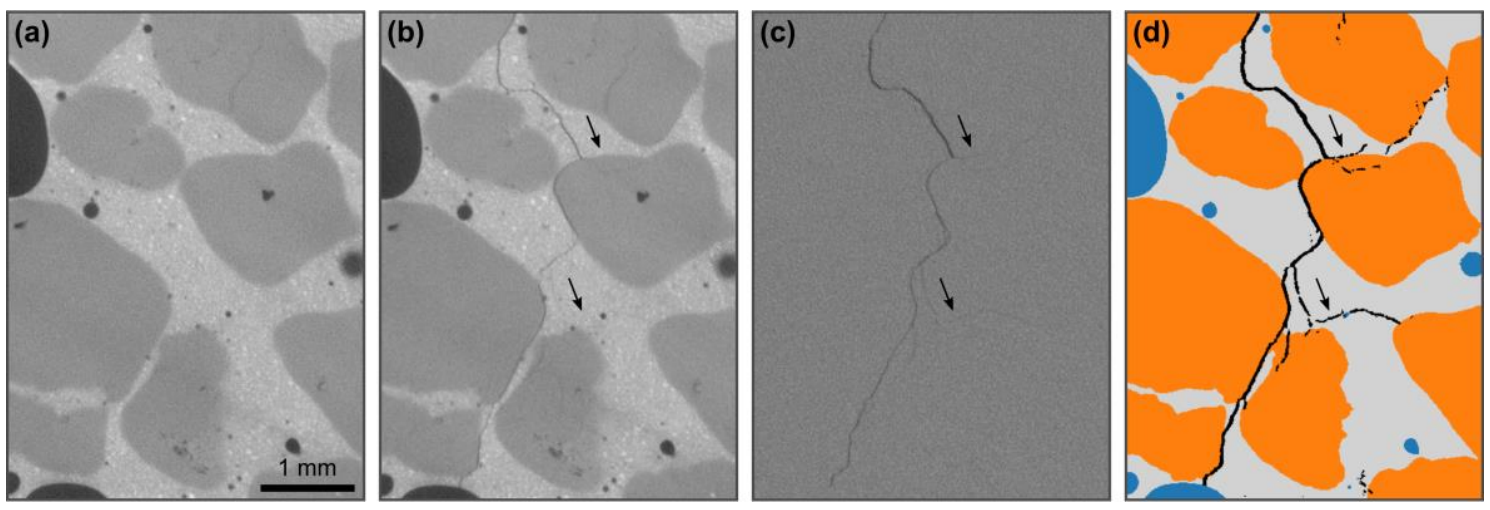

Figure 7: Example of crack detection and segmentation: (a) reference image, (b) deformed image (last loading step, $18 \mathrm{MPa}$ ), (c) subtracted image and (d) segmented crack superimposed on the segmented microstructure (blue: porosity, orange: sand, grey: cement matrix).

Here, a more sophisticated procedure to extract even small cracks in the subtracted image has been used. It is based on a Hessian filtering of the subtracted image. First developed to extract specific features from $3 \mathrm{D}$ medical images ${ }^{[31-33]}$, this type of filter analyses the eigenvalues of the standard second-derivative matrix - also called Hessian matrix - of the image. In fact, the second-order information in the Hessian matrix makes it possible to distinguish local intensity structures, namely blob-, tube- and sheet-like structures. Cracks, which are here dark sheet-like structures, should then be reflected in a high positive eigenvalue (in the direction orthogonal to the sheet) and two small eigenvalues (in tangential direction). Several filters based on Hessian eigenvalues have been proposed to enhance the local sheetness in 3D images (e.g. ${ }^{[33-35]}$ ). Subtracted images were here processed using the filter defined by ${ }^{[1]}$ whose output is

$$
A(\boldsymbol{X})=\lambda_{3}(\boldsymbol{X})-\left|\lambda_{2}(\boldsymbol{X})\right|-\left|\lambda_{1}(\boldsymbol{X})\right|
$$

The eigenvalues $\lambda_{i}$ of the Hessian matrix are defined as $\lambda_{1} \leq \lambda_{2} \leq \lambda_{3}$. The Hessian matrix is as usual computed with the convolution of the subtracted image with the derivatives of Gaussian kernels to 
smooth out noise effect. The sensitivity of the filter can also be adjusted to the characteristic width of structures through the standard deviation $s$ of the Gaussian kernel. Several kernel sizes can be combined to perform a multiscale analysis (see e.g. ${ }^{[1],}{ }^{[36]}$ ). Here the crack network could be extracted with only one Gaussian scale $(s=2)$. Therefore, no normalization of the filtered image (such as that used in ${ }^{[1]}$ ) was necessary.

Cracks are then extracted by thresholding the filtered subtracted image. Most of extracted voxels belong to cracks, but some false detections may remain. We can distinguish false detections due to random XRCT artefacts and those due to transformation errors. The first are far more pronounced at the ends of the specimen because of a slight cone beam effect. Therefore, the first and last 75 slices (i.e. $750 \mu \mathrm{m}$ ) were excluded from the image analysis after visually checking that they did not contain cracks. False detections caused by local transformation errors are typically located between large and very close pores where only a small cement interface remains. The local transformation is then unlikely to be well estimated because of very few exploitable correlation points. Since the location of such false detections is related to the microstructure, they are mostly reproducible from one loading step to another. Therefore, all the detections from the three first loading steps (except one small crack clearly identified at step \#3) were assimilated to false detections and were removed from all other steps, after a morphologic dilation. Finally, connected components smaller than 4000 voxels were also removed. This procedure erases most of the false detections of the crack segmentation. The remaining few are easily discernible from the crack path which is clearly visible.

The obtained cracks segmented from the subtracted image in Figure $7 \mathrm{c}$ are presented in Figure $7 \mathrm{~d}$ where they are superimposed on the segmented microstructure. The latter has been obtained by processing the reference image using total-variation denoising ${ }^{[37]}$, global grey level thresholding and several classical morphological and logical operations. The boundary of the sample section has been detected using the Circle Hough Transform ${ }^{[38]}$, and has also been used to restrict crack detection within the sample. Figure $7 \mathrm{~d}$ shows that the full procedure (DVC-based subtraction and segmentation) makes it possible to detect and extract not only the cracks observed qualitatively in the deformed image, but also some others that are not visible, both in plain constituents - cement or sand grains - and at interfaces.

Note that reference and subtracted images have been processed using Python, relying on NumPy, SciPy and scikit-image packages ${ }^{[39]}$.

\subsection{Location and propagation}

Images acquired at each loading step have been analysed using the full procedure presented above. In addition to the crack detection, the axial strain component has been computed from DVC data, using the displacements of a set of points located on two planes near the ends of the sample, between the reference and the considered configuration. They are used as the nodal displacements of a finite element mesh, made of 8-node elements, covering almost the whole specimen. The average strain of this mesh computed with standard integration procedures provides the overall strain of the sample (see also ${ }^{[20]}$ ). The obtained macroscopic stress-axial strain curve is plotted in Figure 8. It also shows the relative evolution of the total volume of segmented cracks. 


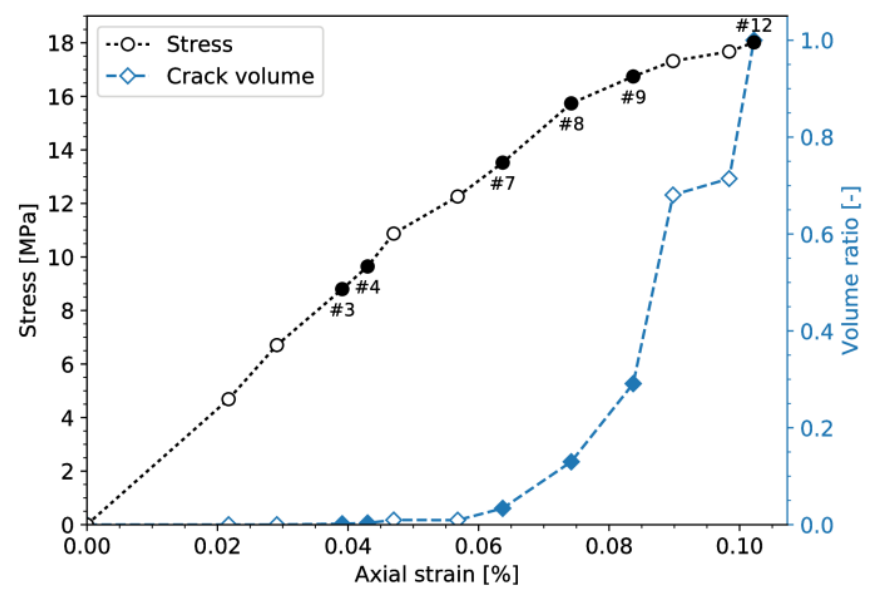

Figure 8: Macroscopic compressive behaviour of the lightweight concrete sample obtained with the in situ device (each point corresponding to an interruption for the XRCT acquisitions) and evolution of global crack volume, normalised with respect to its maximum value. The numbers of the loading steps that will be illustrated in following figures are reported (solid markers).
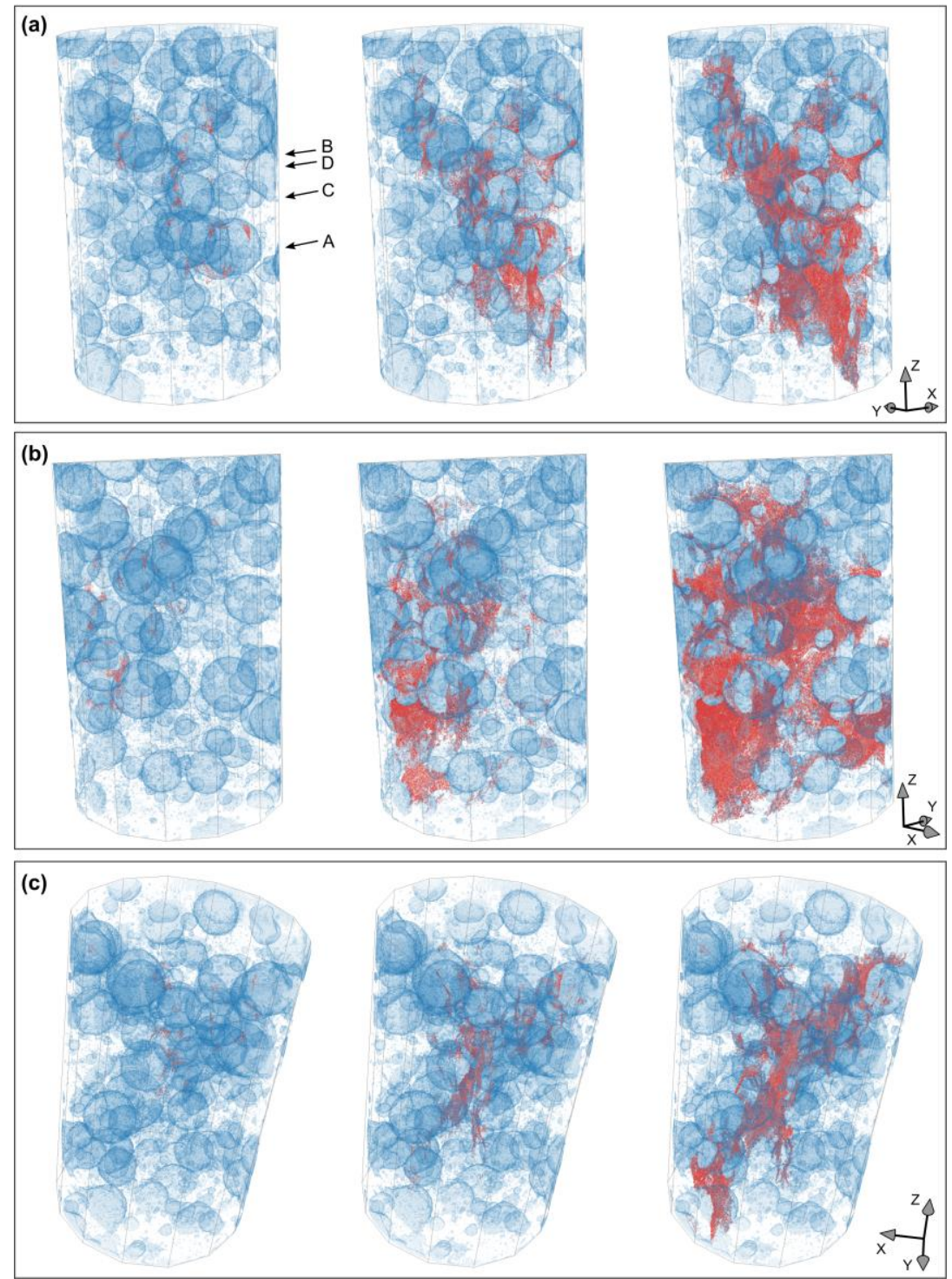

Figure 9: $3 \mathrm{D}$ views of the crack network (red) propagation through the segmented porosity (blue) at loading steps \#7 (13.5 MPa), \#9 (16.7 MPa) and \#12 (18 MPa). (a)-(b)-(c) provide three different points of view. A-B-C-D in (a) refer to the location of the slices presented in Figure 10, Figure 11 and Figure 12. 
In order to limit the effect of the few residual false detections, especially at low loads, an additional image processing has been performed on the segmented cracks. Connected components smaller than 10000 voxels have been removed from the crack network detected at the last loading step (\#12, $18 \mathrm{MPa}$ ). Assuming that cracks did not close during the loading, all voxels detected at all previous steps which did not belong to this last crack network have been excluded. Note that such processing has only been used for global measurement and visualization (Figure 8 and Figure 9). It should also be mentioned that the obtained volume does not directly reflect the opening of crack, notably because the interpolation in the subtraction procedure and the Hessian filter might enlarge thin cracks. Following the method introduced in section 3.2.3, analysing grey levels corresponding to cracks in the subtracted images would be more relevant to estimate crack opening. Nevertheless, the volume depicted in Figure 8 is a qualitative indicator of the overall progression, including propagation and opening, of the crack network. The evolution of cracking in the whole sample is also presented in Figure 9 using 3D views.

The first two microcracks to be detected were observed at relatively low loads (steps \#3 and \#4, 9 MPa). As shown by the crack and microstructure segmentations presented in Figure 10, both cracks initiated from porosity, and more specifically in the cement paste located at the interface of two connected pores. These cracks are barely visible in the XRCT images and it would not have been possible to segment them, or even detect them, without the DVC-based subtraction. Both zones are located far from the ends of the sample which confirms that the specific sample setup was effective to avoid crack initiation caused by imperfect boundary conditions. Two more microcracks were detected in cement paste at step \#5 (11 $\mathrm{MPa}$ ), also at the interface of two close pores. This suggests that these regions, characterised by local narrowing of cement paste between pores, are weakest and/or undergo strain localisation because of the microstructure morphology. The crack volume started to increase significantly when the macroscopic curve deviates from linearity (steps \#6-\#7, 13 MPa, see Figure 8). The crack network is then still composed of several individual microcracks (see Figure 9). Next, they tend to coalesce and to form one main crack as it can be observed at step \#9 ( 17 MPa). The last loading phases are mainly characterised by the propagation and the opening of the crack network, where at least one main propagation plane can be identified (see step \#12 in Figure 9).
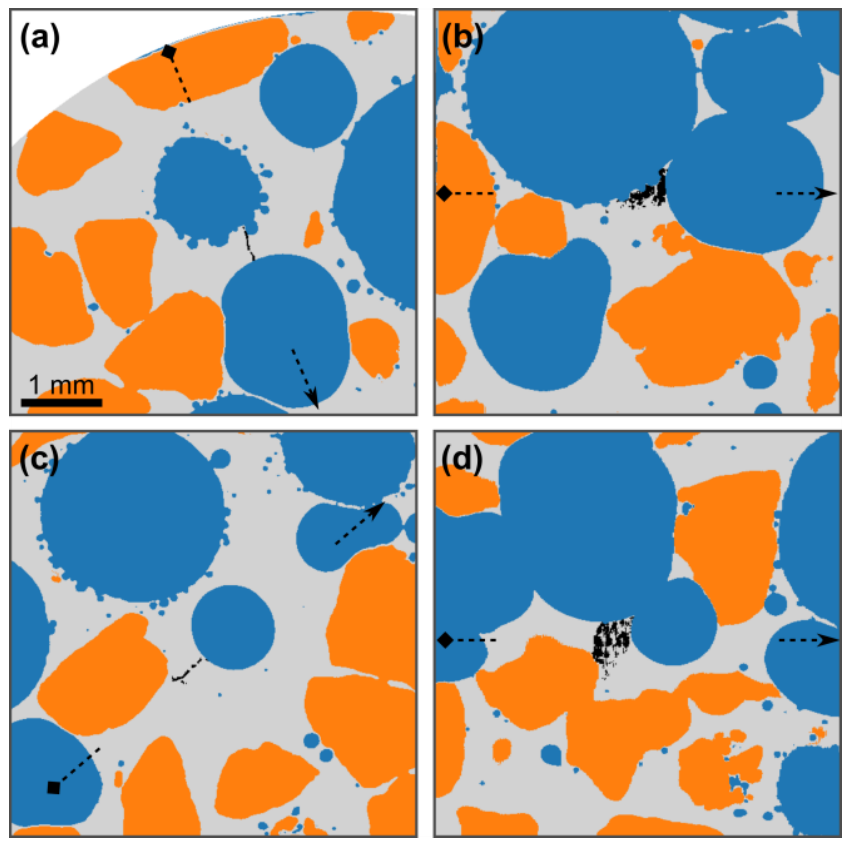

Figure 10: Initiation of cracking at step \#3 - transverse slice A (a) and corresponding longitudinal slice (b) - and step \#4 - transverse slice B (c) and corresponding longitudinal slice (d). The position of longitudinal slices is indicated in the corresponding transverse slices by the dotted arrows (and conversely). The axial location of transverse slices is indicated in Figure 9a. 
Two transverse slices have been chosen to observe more locally the crack propagation and are presented in Figure 11 and Figure 12 (their axial positions are given in Figure 9a). It appears that cracks tend to link the largest pores. Even though they mostly propagate in the cement matrix and at cement/sand grain interfaces, they can also go through sand grains. Figure 11c shows also that the proposed image processing makes it possible to extract complex crack shapes. Finally, a noteworthy morphology of the crack network is shown in Figure 12, where three crack surfaces are observed to propagate separated by approximate angles of $120^{\circ}$, from the bottom of a spherical pore (EPS bead), which is located just above another spherical pore. This typical cracking morphology has already been observed in lightweight plaster under compression and captured by numerical simulation in ${ }^{[1]}$.

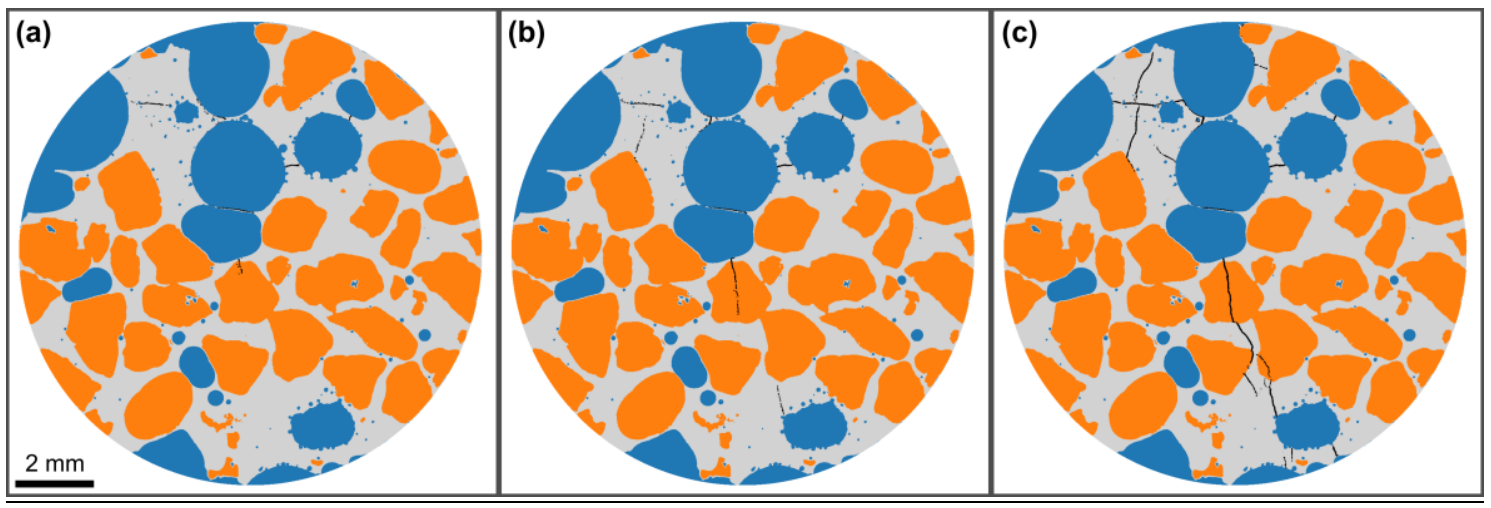

Figure 11: Crack propagation in transverse slice C (see Figure 9a) at loading steps \#8 (a), \#9 (b) and \#12 (c) (the crack observed at the centre of the slices between the two closest pores is a false detection).

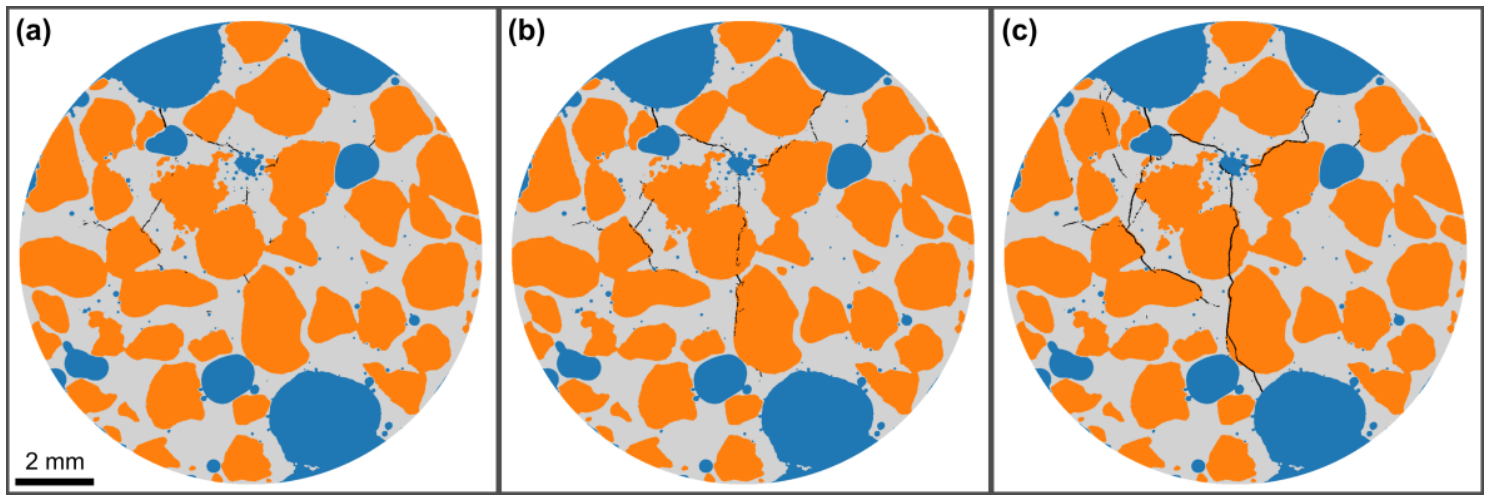

Figure 12: Crack propagation in transverse slice D (see Figure 9a) at loading steps \#8 (a), \#9 (b) and \#12 (c).

\section{Conclusion}

A method based on DVC has been developed to detect and extract microcracks that appear in XRCT images of heterogeneous materials under in situ loading. The general principle consists in subtracting the image of the reference stage to the image of a given loading stage. To do so, the transformation that links both configurations is estimated in a multitude of positions using local DVC routines. It is then densely extended throughout the whole sample, by means of an adjusted interpolation/smoothing procedure that also filters DVC measurements errors. The subtracted image is finally computed after transforming the deformed image back to the same frame as the reference image.

This procedure has been used to detect cracking that progressively developed in a lightweight concrete sample under uniaxial compression. XRCT images have been acquired at several loading steps using a dedicated in situ set-up. In order to overcome a too low local contrast in porosity and sand grains, the DVC routines have been run on positions in cement matrix only, defined according to a grid adapted to 
the microstructure. The image subtraction has been performed for all loading steps and revealed microcracks created by the compression load, even in their early age, with a detection accuracy estimated to a tenth voxel. Some tiny detected cracks were not visible on the XRCT images and would not have been noticed without the DVC-assisted subtraction. Furthermore, the uniform grey level background of subtracted images makes much easier the segmentation of the crack network. A Hessian based filter has been used to segment even the tiniest cracks with a signal similar to the background noise. It is thus possible to characterise the evolution of the complex crack network, from early-age cracking to the propagation through the whole sample. The cracks can be directly located in the undamaged microstructure and easily compared for all loading steps, since all images are transformed back to the reference image frame. In particular, it can be observed that the first cracks initiate from pores, either in cement paste located near the interface of closely connected pores, or at the poles of large quasi-spherical pores. The coalescence of several microcracks results then in a main crack network which tends to connect the largest pores. Its propagation involves different fracture mechanisms, that are cement matrix cracking, sand grain interfacial debonding and sand grain cracking.

Such results are of course useful for a better understanding of the material behaviour and could be compared directly and qualitatively to numerical simulations on realistic microstructures. Future development will aim at get a more quantitative characterisation of cracking, especially regarding to crack opening by analysing the grey levels of the cracks in the subtracted image. This procedure can also be used to investigate evolving damage and fracture mechanisms in various heterogeneous materials, as soon as XRCT images characteristics are suitable for DVC, at least in some parts of the images.

\section{Acknowledgement}

This work has benefited from a French government grant managed by ANR within the frame of the national program Investments for the Future ANR-11-LABX-022-01. The acquisition of the microtomography device of Laboratoire Navier (ENPC) has been made possible thanks to grants from the Region Ile de France (SESAME 2007 program) and CNRS. The contribution of the technical team of LMS (Ecole Polytechnique), and in particular V. de Greef, to the design and the manufacturing of the in situ testing device used to perform the compression tests is acknowledged, together with its funding by the "Matinex" joint program of CNRS and CEA. J. Yvonnet also thanks IUF (Institut Universitaire de France) for its financial support. Finally, the authors thank Prof K. Miled (ENIT, Tunis) for providing lightweight concrete samples and S. Brisard for his helpful suggestions related to image processing.

\section{References}

[1] T. T. Nguyen, J. Yvonnet, M. Bornert, C. Chateau, J. Mech. Phys. Solids 2016, 95, 320.

[2] S. R. Stock, Int. Mater. Rev. 2008, 53(3), 129.

[3] E. Maire, P. J. Withers, Int. Mater. Rev. 2014, 59(1), 1

[4] J. Y. Buffiere, E. Maire, J. Adrien, J. P. Masse, E. Boller, Exp. Mech. 2010, 50(3), 289.

[5] E. N. Landis, T. Zhang, E. N. Nagy, G. Nagy, W. R. Franklin, Mater. Struct. 2007, 40(4), 357.

[6] K. Wan, X. Xue, Mater. Charact. 2013, 82, 32.

[7] S. C. de Wolski, J. E. Bolander, E. N. Landis, Exp. Mech. 2014, 54(7), 1227.

[8] C. Poinard, E. Piotrowska, Y. Malecot, L. Daudeville, E. N. Landis, Eur. J. Environ. Civ. Eng. 2012, 16(sup1), s115-s136.

[9] Skarzyński, J. Tejchman, Strain 2016, 52(1), 26-45.

[10] O. Paetsch, D. Baum, K. Ehrig, D. Meinel, S. Prohaska, in Proceedings of Conference on Industrial Computed Tomography 2012, 321.

[11] M. Voorn, U. Exner, A. Rath, Comput. Geosci. 2013, 57, 44.

[12] H. Deng, J. P. Fitts, C. A. Peters, Comput. Geosci. 2016, 231. 
[13] M. Bornert, F. Hild, J.-J. Orteu, S. Roux, "Digital Image Correlation," in Full-Field Measurements and Identification in Solid Mechanics, M. Grediac and F. Hild, Eds. Wiley-ISTE, 2012.

[14] F. Hild, A. Bouterf, S. Roux, Int. J. Fract. 2015, 191(1-2), 77.

[15] J. Rannou, N. Limodin, J. Réthoré, A. Gravouil, W. Ludwig, M.-C. Baïetto-Dubourg, J.-Y. Buffière, A. Combescure, F. Hild, S. Roux, Comput. Methods Appl. Mech. Eng. 2010, 199(2122), 1307.

[16] J. Lachambre, J. Réthoré, A. Weck, J. Y. Buffiere, Int. J. Fatigue 2015, 71, 3.

[17] L. Wang, N. Limodin, A. El Bartali, J. F. Witz, R. Seghir, J. Y. Buffiere, E. Charkaluk, Mater. Sci. Eng. A 2016, 673, 362.

[18] F. Hild, S. Roux, D. Bernard, G. Hauss, M. Rebai, in VIII International Conference on Fracture Mechanics of Concrete and Concrete Structures (FraMCoS-8), 2013.

[19] M. Bornert, J. M. Chaix, P. Doumalin, J. C. Dupré, T. Fournel, D. Jeulin, E. Maire, M. Moreaud, H. Moulinec, Instrumentation, Mes. Métrologie 2004, 4(3-4), 43.

[20] N. Lenoir, M. Bornert, J. Desrues, P. Bésuelle, G. Viggiani, Strain 2007, 43, 193.

[21] K. Miled, K. Sab, R. Le Roy, Mech. Mater. 2007, 39(3), 222.

[22] K. Miled, O. Limam, Mech. Res. Commun. 2016, 76, 96.

[23] N. Liu, B. Chen, Constr. Build. Mater. 2014, 68, 227.

[24] T. T. Nguyen, "Modeling of complex microcracking in cement based materials by combining numerical simulations based on a phase-field method and experimental 3D imaging", PhD Thesis, Université Paris-Est, 2015.

[25] L. A. Feldkamp, L. C. Davis, J. W. Kress, J. Opt. Soc. Am. A-Optics Image Sci. Vis. 1984, 1(6), 612.

[26] B. K. Bay, T. S. Smith, D. P. Fyhrie, M. Saad, Exp. Mech. 1999, 39(3), 217.

[27] M. Bornert, F. Brémand, P. Doumalin, J.-C. Dupré, M. Fazzini, M. Grédiac, F. Hild, S. Mistou, J. Molimard, J.-J. Orteu, L. Robert, Y. Surrel, P. Vacher, B. Wattrisse, Exp. Mech. 2009, 49(3), 353.

[28] J. Kopp, Int. J. Mod. Phys. C 2008, 19(3), 13.

[29] F. Amiot, M. Bornert, P. Doumalin, J. C. Dupré, M. Fazzini, J. J. Orteu, C. Poilâne, L. Robert, R. Rotinat, E. Toussaint, B. Wattrisse, J. S. Wienin, Strain 2013, 49(6), 483.

[30] J. Dautriat, M. Bornert, N. Gland, A. Dimanov, J. Raphanel, Tectonophysics 2011, 503(1-2), 100.

[31] C. Lorenz, I. Carlsen, T. Buzug, CVRMed-MRCAS'97 1997, 1205, 233.

[32] A. F. Frangi, W. J. Niessen, K. L. Vincken, M. A. Viergever, Medial Image Comput. Comput. Invervention - MICCAI'98. Lect. Notes Comput. Sci. 1998, 1496, 130.

[33] Y. Sato, C.-F. Westin, A. Bhalerao, S. Nakajima, N. Shiraga, S. Tamura, R. Kikinis, Vis. Comput. Graph. IEEE Trans. 2000, 6(2), 160.

[34] M. Descoteaux, M. Audette, K. Chinzei, K. Siddiqi, in Medical Image Computing and ComputerAssisted Intervention - MICCAI 2005 SE - 2 2005, 3749, 9.

[35] K. Ehrig, J. Goebbels, D. Meinel, O. Paetsch, S. Prohaska, V. Zobel, in International Symposium on Digital Industrial Radiology and Computed Tomography, 2011.

[36] Y. Sato, S. Nakajima, N. Shiraga, H. Atsumi, S. Yoshida, T. Koller, G. Gerig, R. Kikinis, Med. Image Anal. 1998, 2(2), 143.

[37] A. Chambolle, J. Math. Imaging Vis. 2004, 20(1-2), 89.

[38] S. Brisard, "Orientation correlations among rice grains, part 4: defining the ROI," Personal communication, http://sbrisard.github.io/.

[39] S. van der Walt, J. L. Schönberger, J. Nunez-Iglesias, F. Boulogne, J. D. Warner, N. Yager, E. Gouillart, T. Yu, and the scikit-image contributors, PeerJ 2014, 2. 